\title{
TREATMENT OF ADHD IN NEUROFIBROMATOSIS TYPE 1
}

The cognitive and behavioral problems and effects of treatment of attention deficit hyperactivity disorder (ADHD) in 20 children with NF1 and ADHD were compared to 26 control children with NF1, 14 controls with ADHD, and 14 controls with normal development, studied at Klinikum Nord Ochsenzoll. Hamburg, Germany. Of 93 children with NF1 followed at the clinic, 46 had ADHD. Among the 4 groups, children with NF1 and ADHD had the lowest IQ scores. Tests of Variables of Attention (TOVA) scores were lower in the NF1/ADHD and ADHD control groups than in the non-ADHD groups. Scores on the Child Behavior Checklist (CBCL) were significantly poorer for the NF1/ADHD group than the NF1 control group. Low dose (5-15 mg) methylphenidate administered to the NF1/ADHD group significantly improved TOVA scores and CBCL scores. (Mautner V-F, Kluwe L, Thakker SD, Leark RA. Treatment of ADHD in neurofibromatosis type 1 . Dev Med Child Neurol March 2002;44:164-170). (Respond: Victor-F Mautner MD, Department of Neurology, Klinikum Nord Ochsenzoll, Langenhorner Chaussee 560, D-22419 Hamburg, (rermany).

COMMENT. NF1 is associated with a $50 \%$ incidence of ADHD, and learning and social problems in children with NF1 are correlated with ADHD. Stimulant medication in children with NF1/ADHD may improve their cognitive, attention, impulsive, and social problems.

\section{TRENDS AND FREQUENCY OF CLONIDINE TOXICITY REPORTED TO POISON CENTER}

Pediatric clonidine exposures were evaluated in 10060 cases reported to the American Association of Poison Control Center's database from Jan 1, 1993 through Dec 31, 1999, at the University of Maryland School of Pharmacy, Baltimore. Patients were followed until their outcome was known. Most exposures (57\%) occurred in children younger than 6 years, 34\% for children between 6 and 12 years, and $9 \%$ for adolescents between 13 and 18 years old. The prevalence of exposures increased 2.5 times between 1993 and 1999. Clonidine had been prescribed as a medication in $35 \%$ of exposures in 6-through 12 year-olds, in $10 \%$ of children younger than 6 years, and in $26 \%$ of adolescents. The proportion of exposures involving a child's medication increased over 7 years. Unintentional overdose was the cause of toxicity most commonly in children younger than 6 years, therapeutic errors predominated in 6-12 year olds, and suicide attempts in adolescents. Sixty percent of cases of clonidine exposure were symptomatic. Of these, lethargy occurred in $80 \%$, bradycardia in $17 \%$, hypotension $(15 \%)$, and respiratory depression (5\%). Only one resulted in fatality, in a 23 -month-old; $40 \%$ exposures had no toxic effect, $39 \%$ were minor toxicities, $19 \%$ moderate, and $2 \%$ major. The evidence of toxic effects occurring in children receiving clonidine for therapeutic reasons, eg ADHD and Tourette syndrome, was of particular concern. (Klein-Schwartz W. Trends and toxic effects from pediatric clonidine exposures. Arch Pediatr Adolesc Med April 2002;156:392-396). (Respond: Wendy Klein-Schwartz Pharm D, MPH, Maryland Poison Center, Department of Pharmacy Practice and Science, University of Maryland School of Pharmacy, 20 N Pine St, Baltimore, MD 21201).

COMMENT. Clonidine is a second-line medication for ADHD, especially with sleep problems, comorbid oppositional defiance disorder, or tics. Except when careful monitoring is possible, combined clonidine and stimulants are not recommended. Avoidance of unintentional overdose or dosage error is important. 